e-Phaïstos

e-Phaïstos

Revue d'histoire des techniques / Journal of the history

of technology

I-1 | 2012

Le patrimoine maritime

\title{
Les murs à palisser « à la Montreuil »
}

Ivan LAFARGE

URL : http://journals.openedition.org/ephaistos/288

DOI : 10.4000/ephaistos.288

ISSN : 2552-0741

Éditeur

IHMC - Institut d'histoire moderne et contemporaine (UMR 8066)

Édition imprimée

Date de publication : 1 juin 2012

Pagination : 79-87

ISSN : 2262-7340

Référence électronique

Ivan LAFARGE, «Les murs à palisser « à la Montreuil » », e-Phaïstos [En ligne], I-1 | 2012, mis en ligne le 01 janvier 2015, consulté le 30 avril 2019. URL : http://journals.openedition.org/ephaistos/288 ; DOI :

10.4000/ephaistos.288 


\title{
Les murs à palisser "à la Montreuil"
}

\author{
Ivan Lafarge, archéologue \\ Bureau de l'archéologie du Département de \\ la Seine-Saint-Denis, \\ doctorant au Centre d'Histoire des \\ Techniques (CH2ST/EA127), \\ Université Paris 1 Panthéon-Sorbonne
}

Le système de culture dit "à la Montreuil" est un mode de production horticole où les arbres fruitiers sont palissés ${ }^{1}$ sur des supports maçonnés (fig. 1).

Il apparaît au XVII ${ }^{\mathrm{e}}$ siècle ${ }^{2}$ et s'est développé jusqu'au $\mathrm{XX}^{\mathrm{e}}$ siècle au nord-est de Paris, sur les terroirs des communes de Montreuil, Bagnolet et Rosnysous-Bois. Les productions liées à ce mode de culture sont essentiellement des pêchers et des pommiers, mais aussi poiriers, cerisiers et vigne. Cette dernière culture était une des productions majeures de Montreuil avant le développement du système des murs à pêches et gardera longtemps son importance au sein d'un système de production qui reste marqué par la polyculture, même si ce sont les pêches qui ont fait la renommée horticole du lieu. S’y associent souvent, au milieu des clos, des groseilliers, fraisiers, légumes et fleurs (surtout depuis la fin du XIX ${ }^{\mathrm{e}}$ siècle) ainsi que quelques arbres fruitiers de plein vent.

Les murs à pêches de Montreuil constituent une originalité de cette localité et ils ont été un des moteurs du développement économique de cette commune pendant l'époque moderne. Leur très important développement au cours du $\mathrm{XIX}^{\mathrm{e}}$ siècle (environ 6ookm linéaires développés sur près de 70oha) a très fortement marqué le paysage (fig. 3).

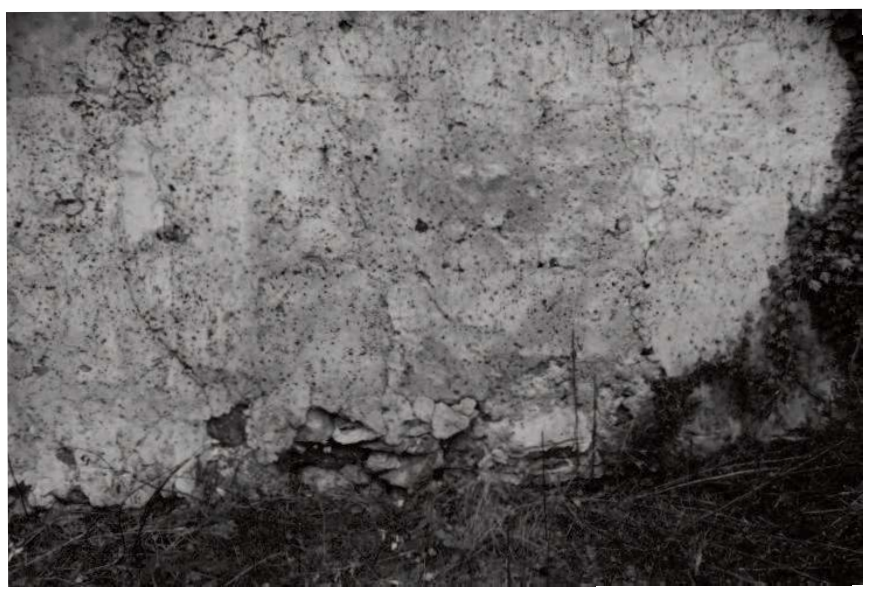

Fig. 1 : Empreinte sur l'enduit d'un arbre palissé. Montreuil, quartier Saint-Antoine

(C) Département de la Seine-Saint-Denis, bureau de l'archéologie, photo Ivan Lafarge). 

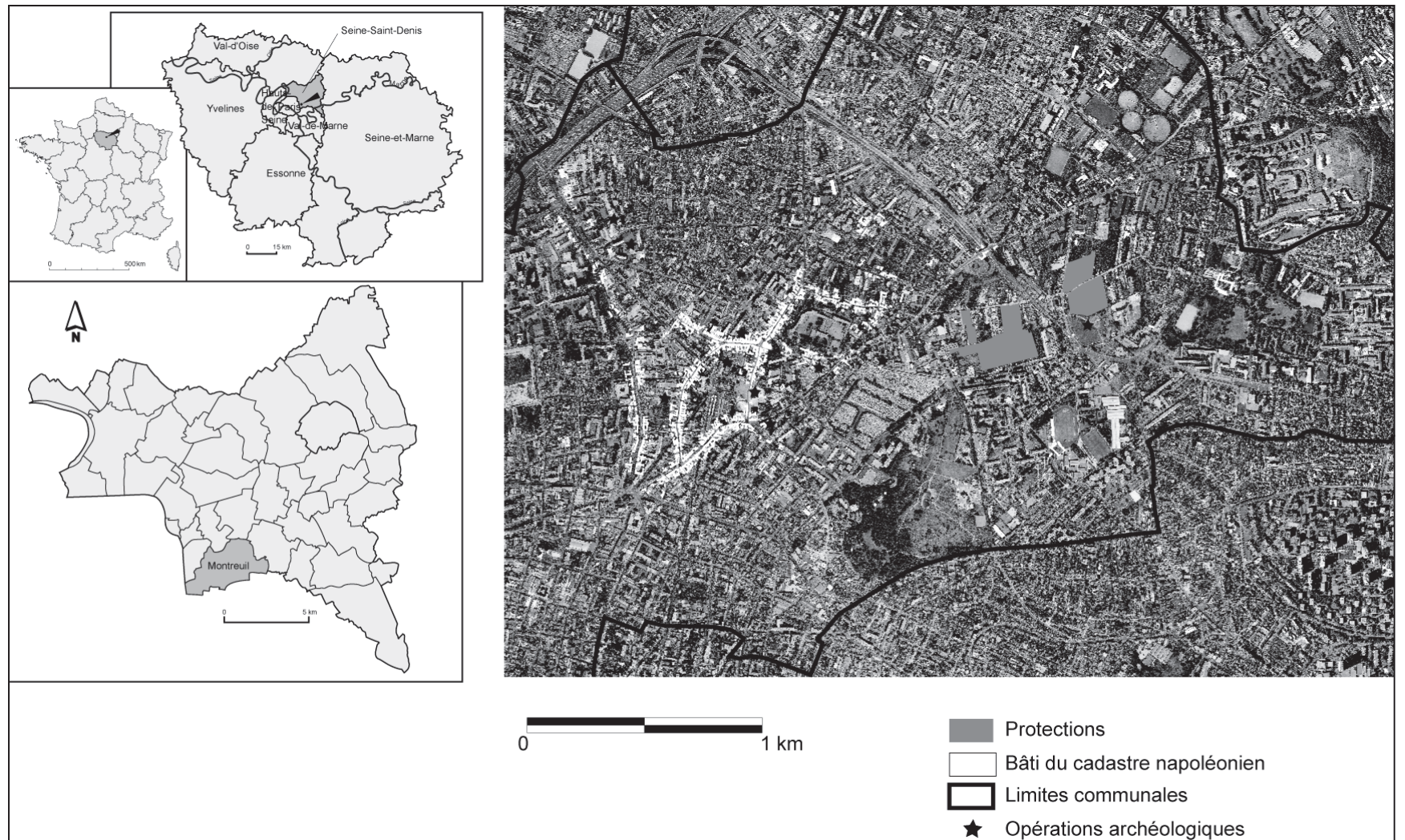

Fig. 2 : Localisation de Montreuil et des zones de mur à pêches protégées au titre de la loi du o2 mai 1930 relative à la protection des monuments naturels et des sites de caractère artistique, historique, scientifique, légendaire ou pittoresque

(C) Département de la Seine-Saint-Denis, bureau de l'archéologie).

À l'époque médiévale, le terroir est essentiellement utilisé pour la culture de la vigne $(55,8 \%$ des terres à la fin du Moyen-Âge) et des céréales (32,2\% des terres à la fin du Moyen-Âge), les bois et zones humides ne représentant que $12 \%$ du terroir à la fin du Moyen-Âge ${ }^{3}$. La culture de la pêche devient prédominante à partir du XVII ${ }^{\mathrm{e}}$ siècle, et supplante progressivement la vigne et les autres arbres fruitiers au XIX ${ }^{\mathrm{e}}$ siècle. Cependant la vigne garde une place importante tant pour la production de vin que pour la table. D'ailleurs, il était encore de tradition au milieu du $\mathrm{XX}^{\mathrm{e}}$ siècle d'utiliser des feuilles de vigne pour l'emballage des pêches.
Les murs à pêches présentent un paradoxe de taille qu'il est nécessaire d'appréhender si on veut les comprendre : malgré l'aspect monumental qui se dégage de l'ensemble des clos, leur rôle n'est pas constructif ni même parcellaire, mais horticole ; leur fonction n'est que le palissage des arbres et leur mode de mise en œuvre est en lien direct avec cette finalité. Ils jouent un rôle essentiel dans la production des fruits, et sont en tant que tels des objets techniques.

L'originalité de ce système horticole et la période de son essor font que ce système a été l'objet de nombreuses publications dans la littérature agronomique 
depuis le XVIII ${ }^{\mathrm{e}}$ siècle ${ }^{4}$, le plus souvent du point de vue des techniques culturales. Dans ces productions, il est parfois difficile de trouver des descriptions des murs à palisser "à la Montreuil" cohérentes entre elles. Cette variabilité des discours s'avère en fait assez cohérente avec la variabilité des formes observables sur le terrain.

Près d'une dizaine d'opérations d'archéologie préventive menées au cours de la dernière décennie permettent, à l'échelle du terroir montreuillois, de commencer à comprendre les modes de construction qui ont présidé à l'édification de ces murs. À défaut d'être exhaustive, l'analyse archéologique de ces constructions permet de comprendre le fonctionnement de ce système de culture, même si les techniques horticoles ${ }^{5}$ en elles-mêmes demandent encore à être documentées.

La plupart des murs à pêches qui ont été observés ne sont pas antérieurs au XVIII ${ }^{\mathrm{e}}$ siècle, toutefois quelques uns ont livré des éléments permettant de les dater du XVII ${ }^{e}$ siècle, voire de la fin du XVI ${ }^{\mathrm{e}}$ siècle $^{6}$.

Dans ces constructions, le plâtre joue un rôle prépondérant et on associe souvent des carrières à leur développement, notamment à Montreuil. Cependant, il ne faut pas s'y tromper : le développement de la production de pêches à Montreuil n'a pas précédé celui de la production de plâtre puisque l'usage de ce matériau prédomine déjà dans la construction au nord-est de Paris dès le Moyen Age ${ }^{7}$. Il n'en demeure pas moins que l'abondance de ce matériau directement accessible sur place a joué un rôle essentiel dans le développement technique de l'horticulture à la Montreuil.

Si le plâtre est exploité très tôt sur les coteaux de Montreuil, ce n'est effectivement qu'à partir de la fin du XVIII ${ }^{\text {e }}$ siècle que cette ressource connait un essor remarquable et que les grandes carrières se développent $^{8}$ (fig. 4), notamment avec des carrières découvertes. Cet essor répond aux besoins constructifs de la première industrialisation. Ces plâtrières génèrent un volume important de déchets qui sont écoulés dans d'autres déclinaisons de la construction ou de l'agriculture : pierres siliceuses des marnes pour la construction ; marnes pour l'amendement des champs, voire la fabrication de chaux ; limons utilisés dans des briquetteries etc... ${ }^{9}$ Ce développement n'empêche pas la production, toujours ponctuelle, de plâtre par les horticulteurs ou, à tout le moins, les maçons, à partir de gypse de récupération ou de gypse directement tiré des parcelles. Si la nécessité de creuser, pour ce faire, des puits à des profondeurs atteignant ou dépassant la dizaine de mètres laisse dubitatif, cette pratique est attestée sous la forme de creusement de fosses d'extraction ponctuelles ou de "décombrement" de carrières et par le fait que les cultivateurs de Montreuil possèdent, dès le début du XVII ${ }^{\mathrm{e}}$ siècle, l'outillage nécessaire au "tirage de pierre"10.

\section{Murs à pêches, une construction normée}

Les murs à pêches de Montreuil sont des constructions relevant de principes qui sont très tôt normés. Cette "normation" se caractérise par le fait que la construction des murs répond toujours à un ensemble de règles générales, sans pour autant que ces règles soient absolues. Ainsi, on retrouve systématiquement dans ces constructions des chaines verticales de plâtre dont le rôle est de rigidifier l'ensemble lié à la terre, des arases de plâtre à hauteurs régulières, des enduits biensûr, et des chaperons en couverture (fig. 4).

De la même manière, les dimensions ont une certaine variabilité, tout en restant dans un ensemble de proportions prédéfinies. Enfin l'orientation même des murs répond à une adéquation entre la topographie du terrain et l'exposition des surfaces de palissage.

\section{Constituants}

L'observation des constituants de maçonnerie des murs à pêches montre clairement que les matériaux dont ils sont constitués sont tous d'origine locale. Pour les murs construits au XIX ${ }^{\mathrm{e}}$ siècle, on observe que les pierres sont en majorité des chailles, silex de qualité médiocre, et des calcaires siliceux, auxquels s'ajoutent différents types de remplois : blocs ou moellons calcaires, plâtras. Le fait qu'il s'agisse de matériaux locaux pour leur plus grande part en remploi participe d'un principe d'économie facilement compréhensible, c'est 


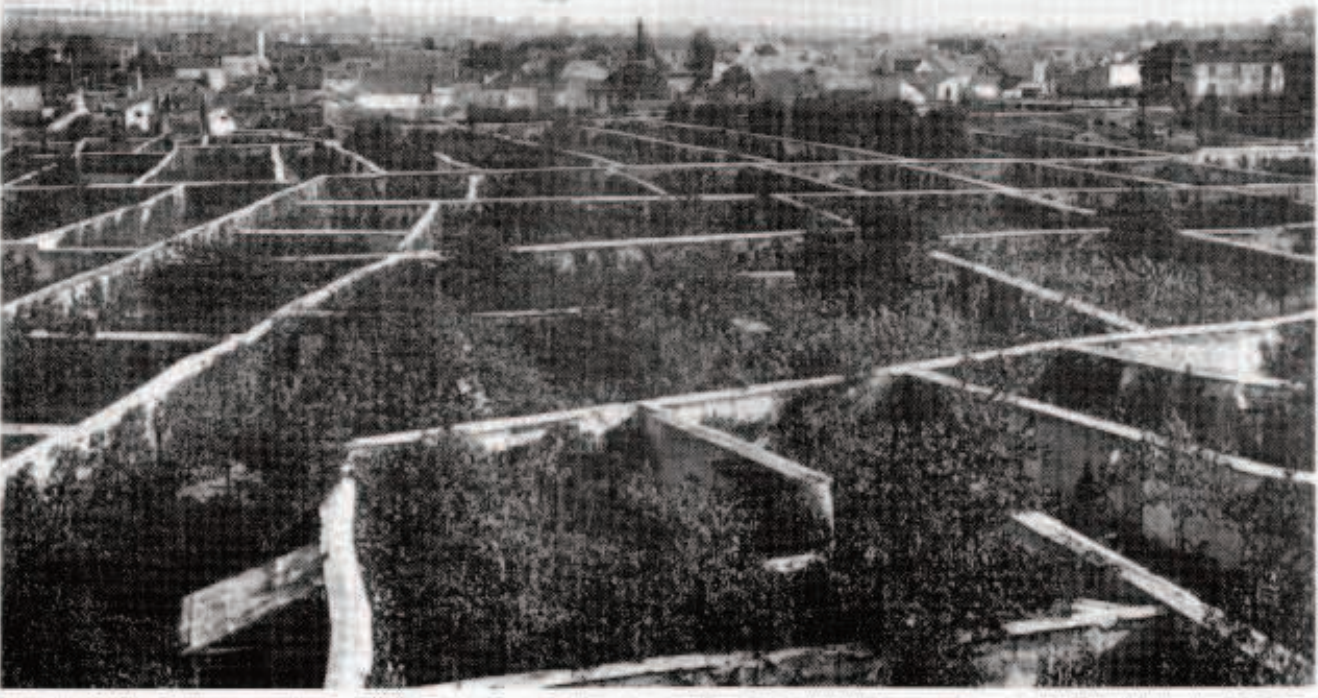

Fig. 3 : Vue des clos à pêches dans la première moitié du XX $X^{e}$ siècle (collection Département de la Seine-Saint-Denis, bureau de l'archéologie).

Fig. 4: Carte pos tale, vers 1910 ; au premier plan, une plâtrière, à l'arrière plan, les clos à pêches, puis le village (collection Département de la SeineSaint-Denis, bureau de l'archéologie).

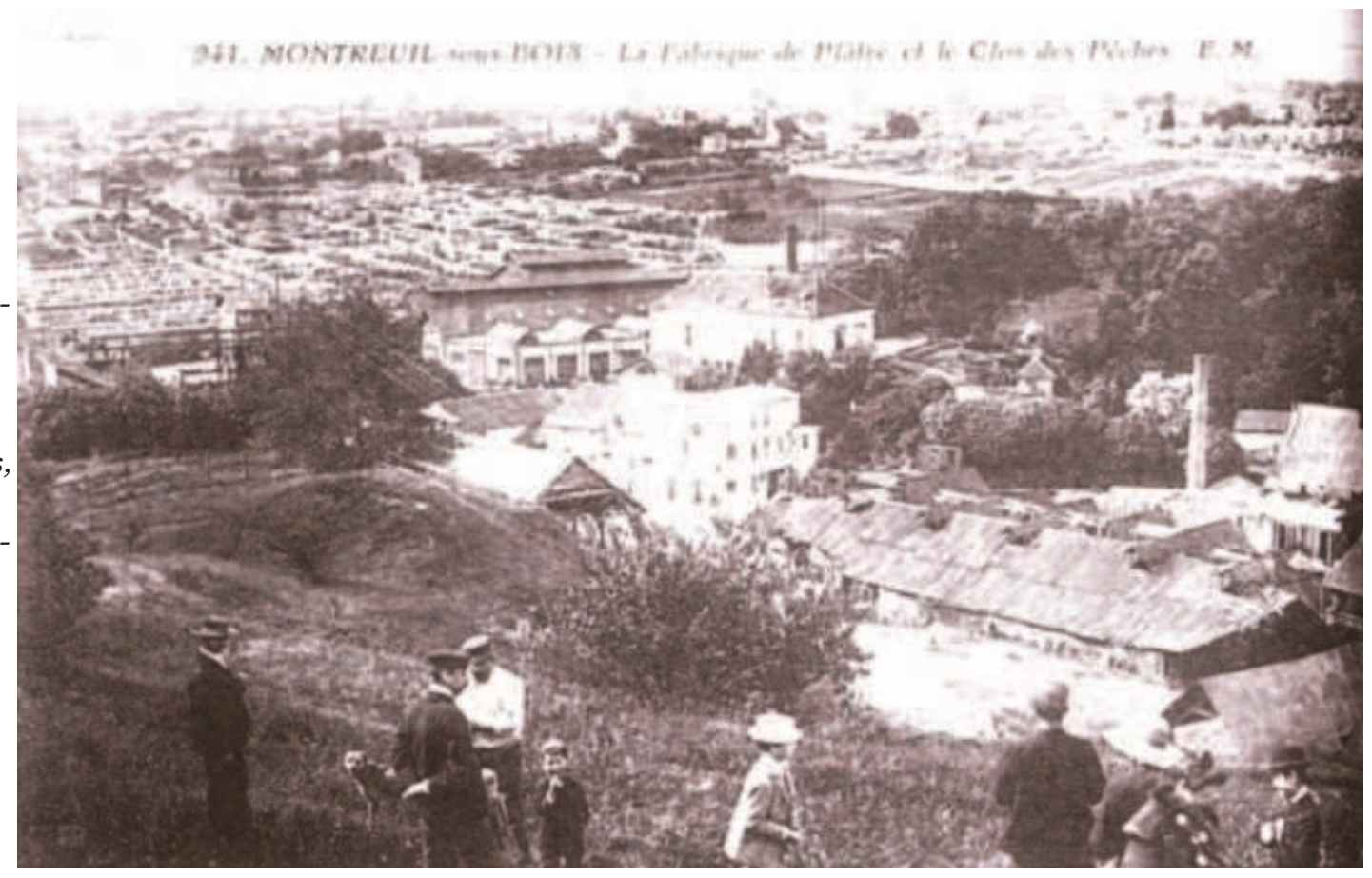


aussi en fait, une conséquence des exploitations de plâtrières.

L’intérêt du plâtre est double : d'une part, il est aisé de s'en procurer à moindre coût ; d'autre part, c'est un matériau souple dans lequel il est possible de planter et déplanter des clous et d'accrocher ou sceller tous les accessoires nécessaires à la production intensive de fruits.

\section{Morphologie}

Ces constructions, peu fondées, sont larges à la base de 40 à $50 \mathrm{~cm}$ (environ 1,5 pieds $^{11}$ ) et hautes en moyenne de $2,70 \mathrm{~m}$ à $3,50 \mathrm{~m}$ ( 8 à 10 pieds, 9 pieds étant la hauteur la plus fréquente). Leur largeur se réduit en hauteur pour atteindre 25 à $35 \mathrm{~cm}$ ( 1 pied maximum) au sommet. Ils sont couverts d'un chaperon, au surplomb de 15 à $16 \mathrm{~cm}$ (soit $1 / 2$ pied, ou 4 pouces) pour une épaisseur de 3 à $5 \mathrm{~cm}$ (1 à 2 pouces). Celui-ci est exclusivement constitué de plâtre jusqu'à la fin du XIX ${ }^{\mathrm{e}}$ siècle où on voit se développer l'usage de tuiles plates de petit moule ; au cours du $\mathrm{XX}^{\mathrm{e}}$ siècle, on voit apparaître des tuiles mécaniques ${ }^{12}$.

Les murs affectent donc un profil légèrement "pyramidal" du fait de leur retrait en élévation et un aspect blanc du fait de leur enduit de plâtre. Ils sont regroupés en clos étroits et communicant entre eux. La largeur moyenne de ces clos est de 8 à 12 mètres (4 à 6 toises), ils sont divisés par des refends, murs formant des divisions dans la longueur des clos, tous les 10 à $20 \mathrm{~m}$ (5 à 10 toises $)^{13}$. Le plus souvent les refends ne s'appuient sur les murs principaux que d'un seul côté, permettant ainsi le passage. Il est important cependant pour en comprendre l'organisation de bien avoir à l'esprit que les murs à palisser ne sont pas des murs parcellaires : si quelques murs peuvent se confondre avec une division parcellaire, celle-ci est rarement leur fonction première. Dans bien des cas, pour de simples raisons de rendement, les murs sont en retrait des limites de parcelles, on les appelle alors des côtières ou costières.

\section{Orientation}

Le but du palissage étant d'augmenter la productivité des arbres, il est logique qu'on ait cherché à construire les murs en fonction de leur meilleur taux d'exposition. À Montreuil, cette recherche se traduit par des expositions préférentielles vers l'est pour les murs principaux et généralement le sud-sud-est pour les refends, en sorte qu'une des deux faces des murs puisse bénéficier d'une constante exposition au soleil. Ainsi les murs principaux sont en général d'orientation nord-sud (nord-nord-est sud-sud-ouest) avec une inclinaison par rapport au nord variant en fonction de la topographie et de l'humidité du terrain ; les refends s'organisent par rapport aux murs longitudinaux en formant des angles entre 45 et $90^{\circ}$, en fonction de la topographie et de l'exposition.

\section{Mise en oeuvre}

La fondation est peu profonde (30 à $50 \mathrm{~cm}$ soit 1 à 1,5 pieds) ; elle est constituée de gros moellons de gypse ou de pierre siliceuse issue du substrat tertiaire local (meulière, ou silex). Elle est liée à la terre. L'élévation est hétérogène, marquée par les remplois approvisionnés localement. On y observe une majorité de pierres issues directement du sol (gypse, meulière, silex tertiaire ou chaille) mâtinée d'éléments remployés (plâtras, pierres diverses, éléments calcaires...) généralement bien triés, on n'observe pas de grès ou de briques avant des réparations contemporaines. Elle est constituée de deux parements adossés et liés par un blocage de terre. L'ensemble est disposé en assises opportunistes (selon les tailles des blocs) au sein desquelles les blocs de plus grande taille sont utilisés en boutisses (ou parpaings) traversant la maçonnerie afin d'en constituer un renfort ponctuel. Quelquefois ces blocs sont remplacés par des os longs de bovidés ${ }^{14}$. Les élévations sont montées à l'aide de coffrages en planches de Lorraine ${ }^{15}$ sans pour autant que la construction ne relève d'un système coffré, le planchage n'a d'autre fonction que de tenir les moellons le temps du séchage de la terre formant le liant. Horizontalement, cette élévation est renforcée régulièrement 


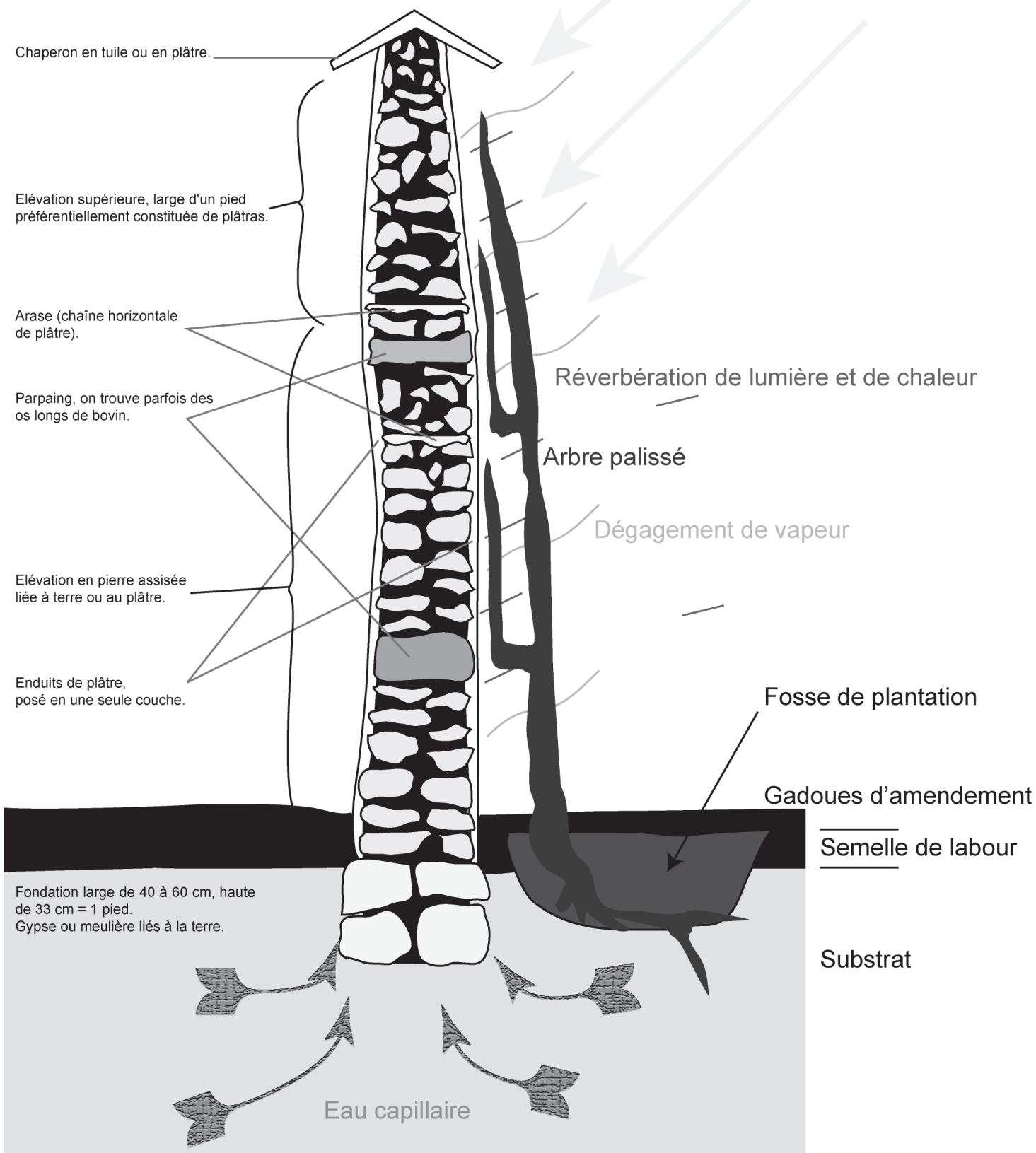

Fig. 5 : Coupe schématique de principe des murs à pêches d'après les observations archéologiques et les descriptions des ouvrages d'horticulture avec schéma fonctionnel

(CDépartement de la Seine-Saint-Denis, bureau de l’archéologie, dessin Ivan Lafarge). 
par des assises de mise à niveau en plâtre, dites "arases", tous les 80 à $90 \mathrm{~cm}$ ( 2,5 à 3 pieds), qui apportent une plus grande cohésion à l'ensemble ; peut-être permettent-elles la construction en plusieurs temps. Ce système est renforcé régulièrement dans toute sa hauteur par des "chaînes" constituées d'une maçonnerie similaire entièrement liée au plâtre, larges d'environ un mètre (environ 3 pieds) au minimum tous les 2 à $3 \mathrm{~m}$ (6 pieds ou 1 toise à 10 pieds).

\begin{abstract}
«Les cultivateurs de Montreuil construisent leurs murs avec le plus de légèreté et le moins de frais qu’il est possible. Ils ne leur donnent, à la base, qu'environ vingt pouces de largeur, et au sommet, qu'un pied. Ils n'emploient généralement que du mortier de terre. Ils forment, de dix pieds en dix pieds, des chaînes en plâtre de trois pieds. Les deux ou trois pieds du sommet sont faits avec des débris de vieux plâtres ; ils couronnent ces murs par un chaperon, avec une saillie de quatre pouces. $»^{16}$.
\end{abstract}

« Lorsqu'à Montreuil on construit un mur pour le garnir de pêchers on le fait, à sa base, épais de 40 centimètres qui au sommet se réduisent à 30 centimètres, et on donne une hauteur de 3 mètres (...) Ces murs doivent être enduits, de chaque côté, d'une couche de plâtre épaisse de 3 centimètres (...) le sommet de ces murs est garni d'un chaperon auquel on donne une saillie de 13 centimètres et $1 / 2$ à l'exposition du levant et de 16 centimètres pour les autres expositions. "17

D'après certaines descriptions, les enduits sont posés au fur et à mesure de la pousse des arbres palissés, cette assertion laisse perplexe dans la mesure où elle implique que les joints restent à la merci des intempéries tant que l'arbre n'a pas poussé. Par ailleurs, il semble que la surface de l'enduit doive être traitée différemment en partie basse -non lissé- et en partie haute -coupé à la truelle pour la respiration de l'enduit, pour réguler les restitutions de chaleur et d'humidité du mur-. Pour autant, la principale difficulté que pose l'observation des enduits est due à leur constante réfection lors de l'utilisation des murs, en effet le palissage provoque la pose et l'enlèvement régulier de clous entraînant sans arrêt des réfections ponctuelles de l'enduit. Ainsi, il nous est apparu à plusieurs reprises déri- soire d'investir le champ d'une étude archéologique des élévations dans leur longueur, celles-ci ayant été systématiquement marquées par l'usage et l'entretien ; clouage, déclouage, réfections d'enduit, réparations de brèches... Si une telle approche permettrait un microphasage en chronologie relative, elle n'apporterait pas d'éléments particuliers ni du point de vue de la chronologie générale, ni du point de vue du fonctionnement des murs.

\section{Principe et mode de fonctionnement (fig.5)}

Les murs ont pour but de créer artificiellement un microclimat favorable au développement des fruits :

- ils réduisent l'action du vent par leur élévation ; ou

- leur disposition sur le terrain naturellement humide, dont le drainage peut être amélioré par défoncement et mise en place de fosses, leur permet de jouer naturellement un rôle de pompage régulier et limité en volume par capillarité. Les matériaux constituant les maçonneries, terre et plâtre ont la capacité de se charger de cette eau capillaire, que l'exposition au soleil permet de restituer sous la forme d'évaporation. À l'inverse, les chailles, meulières et silex sont très imperméables et ne se chargent pas en eau rendant les échanges hygrométriques plus efficaces et limitant les efflorescences de sels (salpêtre).

- ils permettent une augmentation du taux d'hygrométrie au droit des arbres palissés par évaporation lorsque le soleil vient frapper l'enduit ; ou

- ils réverbèrent la lumière et la chaleur du soleil. Associé à ce système, l'engraissement des terrains par les gadoues issues des ordures parisiennes en permet un enrichissement puissant par un épandage généralement annuel, parfois tous les deux, trois, quatre ou cinq ans ${ }^{18}$. Les terrains, rendus acides par cet apport d'engrais doivent régulièrement être amendés par marnage, à intervalles réguliers, mais moins souvent que 
l'engraissement par les gadoues. Là encore, les stériles de carrières jouent un rôle prépondérant, disponibles dans les plâtrières ou directement dans le sous-sol, les marnes locales peuvent suffire.

Les arbres sont plantés à la base des murs auxquels ils sont fixés à mesure de leur croissance par des bandes de tissu clouées sur l'enduit : les loques ${ }^{19}$. Cette disposition permet au gré des échanges thermiques et hydriques de forcer la croissance des arbres et d'en augmenter la productivité. Des portes permettent le passage d'un clos à l'autre et des ruelles peu nombreuses parcourent l'ensemble de leur étendue.

L'essor des murs à pêches pour la production et la vente à grande échelle sur les marchés parisiens puis étrangers est lié au développement de l'exploitation du plâtre d'une part et à la croissance urbaine d'autre part du fait de la réduction des exploitations de plâtrières dans l'enceinte parisienne autour de 1750.

Enfin le plâtre gros, ressource locale, présente la qualité de permettre les échanges de chaleur entre le jour et la nuit, propices à la création d'un microclimat favorable au développement des pêchers aux abords du mur.

Ce développement dont le premier débouché est le marché parisien atteindra toute l'Europe septentrionale au milieu du XIX ${ }^{\mathrm{e}}$ siècle. Il sera mis à mal avec le développement du chemin de fer, la production de pêches, en se repliant sur le marché local, va laisser de nouveau la place aux pommes et aux poires, puis aux fleurs.

Le démarrage à la fin de l'année 2011 d'un premier chantier de restauration de grande ampleur des murs à pêches est l'occasion de se réinterroger sur leur intégration patrimoniale : restaurer un tel objet sans en rétablir l'usage a-t-il un sens?

1 Le palissage est une technique qui consiste à conduire une plante sur une structure, « une muraille ou un treillage » en y attachant les tiges et les branches à l'aide de liens, dans le but d'en améliorer la qualité et le rendement. Cette technique est notamment utilisée dans les domaines de la viticulture et de l'arboriculture. À Montreuil, le palissage est dit « à la loque » parce qu'on utilise des pièces de tissus fixées sur le support, des murs en l'occurence, par des clous, cette méthode est selon l'abbé Schabol (voir bibliographie et note suivante), la meilleure qui soit.

2 SCHABOL Roger (abbé), La théorie et la pratique du jardinage et de l'agriculture, par principes et démontrées d'après la physique des végétaux, Paris $1767, \mathrm{p}$ 322, selon cet auteur cette pratique serait apparue vers 1620 .

3 PESIER S., Montreuil-sous-Bois aux XIVe et XVe siècles. Mémoire de maîtrise sous la direction de Claude Gauvard, Université Paris 1 Panthéon-Sorbonne, Paris, novembre 1994, 173p. ill.

4 À ce sujet, Jean-Jacques Péru fait une présentation assez développée de la bibliographie spécialisée dans le rapport de diagnostic archéologique du 134-142 rue Saint-Antoine : DUFOUR JeanYves, LAFARGE Ivan et PERU Jean-Jacques, Montreuil, 134-142 rue Saint-Antoine, rapport de diagnostic archéologique, 2009. Cette littérature étant très abondante, on ne donne en bibliographie que quelques titres essentiels de manière indicative.

5 Forçage des racines, forme du palissage à la loque, ébourgeonnage, taille...

6 Par exemple : le diagnostic archéologique réalisé par Arnaud Prié sur l'emplacement de l'ancienne ferme Saint-Antoine, d'origine médiévale, s'il n'a pas livré de vestiges justifiant la réalisation d'une fouille, a mis en évidence les fondations de plusieurs murs à palisser et une fosse livrant du mobilier attribué à la fin du XVI siècle, au XVII ${ }^{e}$ siècle puis au XVIII ${ }^{\mathrm{e}}$ siècle (PRIE Arnaud, Montreuil, angle des rues Maurice Bouchor et de la Nouvelle France, Inrap, Centre-Île-de-France, p.31-32). Il s'agit là des éléments les plus anciens mis au jour en relation directe avec des murs à pêches.

7 Le développement des fouilles archéologiques dans la banlieue parisienne, en particulier depuis les années 1990 a permis d'observer l'importance de l'usage du plâtre dans la construction depuis le Moyen-Âge, non seulement à Paris, mais aussi aux alentours et en particulier au nord et au nord-est de la capitale. Pour ne citer qu'un exemple géographiquement cohérent avec les murs à pêches, on mentionnera l'usage du plâtre comme liant dans les fondations et les voûtements, ainsi que pour les parties hautes des murs gouttereaux du choeur de l'église Saint-Pierre-SaintPaul de Montreuil édifié à partir du XIIe siècle et dont l'élévation est à dater dans la décennie 1195-1205.

8 Il semble que l'exploitation du plâtre au Moyen-Âge et au début de l'époque moderne soit moins étendue en banlieue qu'à partir du XVIIIe siècle, le rôle de Paris dans ces exploitations reste prédominant. À Montreuil comme dans de nombreuses localités de la banlieue de Paris, l'exploitation, si elle existe très tôt, reste assez ponctuelle avant le XVIII ${ }^{\mathrm{e}}$ siècle.

9 LAFARGE Ivan (dir.), Une expérience de cuisson du gypse sur les parcelles des murs à pêches (Montreuil, 93) les 16 et 17 juin 2005. Conseil général de Seine-Saint-Denis, DCJS, Service de la Culture, Bureau du patrimoine, Centre d'archéologie, Epinaysur-Seine, 2006, 37 p. 
10 LAFARGE, DUFOUR, PERU, 2009, op. cit., p. 98 à 103

11 Pour la conversion des mesures d'Ancien Régime, on utilise ic le tableau de PORTET Pierre, La mesure de Paris, 2008, p.12.

12 Leur présence n'est pas un indice de datation du mur lui même, mais des dernières réparations liées à son fonctionnement ou à son remploi comme limite parcellaire.

13 Ces mesures sont en réalité assez variables, tous les auteurs donnent des espacements différents, il semble en fait que l'espacement entre les murs soit réglé par la taille de la parcelle et le nombre de clos qu'elle contient. Comme tous les murs sont mentionnés au cadastre napoléonien, une recherche sur les tailles des parcelles nécessiterait un minutieux travail de recoupement et de dépouillement. On retiendra cependant que selon F. Bournon, on peut estimer la présence de 900 à 1000m linéaires de mur par hectare (BOURNON F., Montreuil, État des communes à la fin du XIX ${ }^{\mathrm{e}}$ siècle, 1906).

14 LAFARGE, DUFOUR, PERU 2009, op. cit., p. 79. Il est à noter que l'usage d'os animaux comme tirants ou comme chevilles est assez fréquent dans la maçonnerie de plâtre traditionnelle.

15 AUDUC, Arlette, Montreuil patrimoine horticole - Seine-SaintDenis, collection Itinéraires du patrimoine, ministère de la Culture, inventaire général, 1999, 40 p.

16 MOZARD Jean, Principes pratiques sur la culture des arbres fruitiers et principalement du pêcher, Paris, 1814

17 LEPERE Alexis, Pratique raisonnée de la taille du pêcher principalement en espalier carré, Paris, 1852.

18 LAFARGE, DUFOUR, PERU, 2009, op. cit., p. 86-93

19 Le palissage à la loque, loué par Schabol (op.cit.), permet un forçage efficace des rameaux sans pour autant en blesser la jeune écorce, en revanche, il requiert une entretien régulier.

\section{Bibliographie :}

AUDUC Arlette, Montreuil patrimoine horticole - Seine-SaintDenis, collection Itinéraires du patrimoine, ministère de la Culture, inventaire général, 1999, $40 \mathrm{p}$.

BOURNON Fernand, Montreuil, État des communes à la fin du XIX siècle, publié sous les auspices du Conseil général de la Seine, Montévrain, 1906, $155 \mathrm{p}$.

CERCY Christine, Montreuil, rue Édouard Branly 93.048.oo6 AH, DFS de fouille d'évaluation archéologique, Épinay-sur-Seine, Saint-Denis, 2006, 28 p. ill.

DUFOUR Jean-Yves, Montreuil (Seine-Saint-Denis), 25-31, rue Ernest Savart, Document final de synthèse après évaluation archéologique et ethnographique (21/02/2001 - 12/03/2001), INRAP, Pantin, Saint-Denis 2001, 25 p. ill.

DUFOUR Jean-Yves, Montreuil (Seine-Saint-Denis), rue des bons plants, Document final de synthèses après évaluation archéologique (16/07/2001 - 23/07/2001), INRAP, Pantin, Saint-Denis 2001, 18 p. ill.
DUFOUR Jean-Yves, Montreuil (Seine-Saint-Denis), Ilot Branly-Allende, rapport de diagnostic archéologique (21/12/25004 o6/01/2005), INRAP, Pantin, Saint-Denis 2005, 50 p. ill.

DUFOUR Jean-Yves, « Essai d'archéologie horticole en banlieue parisienne, Saint-Denis et Rueil-Malmaison (XIV ${ }^{\mathrm{e}}-\mathrm{XIX}^{\mathrm{e}}$ siècle) », Histoire et sociétés rurales, $\mathrm{n}^{\circ} 7$, 1er semestre 1997, p. 11-40.

DUFOUR Jean-Yves, LAFARGE Ivan et PERU Jean-Jacques, Montreuil, 134-142 rue Saint-Antoine, rapport de diagnostic archéologique, 2009, $142 \mathrm{p}$.

LAFARGE Ivan (dir.), Une expérience de cuisson du gypse sur les parcelles des murs à pêches (Montreuil, 93) les 16 et 17 juin 2005, Conseil général de Seine-Saint-Denis, DCJS, Service de la Culture, Bureau du patrimoine, Centre d'archéologie, Épinay-sur-Seine, 2006, $37 \mathrm{p}$

LANGLOIS Hippolyte, Le livre de Montreuil aux pêches, théorie et pratique de la culture de ses arbres, Paris, 1876, $497 \mathrm{p}$.

LEPERE Alexis, Pratique raisonnée de la taille du pêcher principalement en espalier carré, 3ème édition, Paris, 1852.

MOZARD Jean, Principes pratiques sur la culture des arbres fruitiers et principalement du pêcher, Paris, 1814, $160 \mathrm{p}$.

PESIER S. - Montreuil-sous-Bois aux XIV et XVe siècles. Mémoire de maîtrise sous la direction de Claude Gauvard, Université de Paris 1 Panthéon-Sorbonne, Paris, Novembre 1994, 173 p. ill.

PESIER S., « Un village d'Île-de-France aux XIV et XV siècles, Montreuil-sous-Bois », Paris et Île-de-France-Mémoires, 1996, tome 47, p. 61-174.

PRIE Arnaud, Montreuil, angle des rues Maurice Bouchor et de la Nouvelle France, Inrap, Centre-Île-de-France, 49 p.

PORTET Pierre, « La mesure de Paris », Manuscrit auteur, 2008, 40 p., publié dans CHARBONNIER Pierre (Éd.) Les anciennes mesures locales du Bassin Parisien et du Nord, d'après les tables de conversion [http://halshs.archives-ouvertes.fr/docs/oo/13/50/ 71/PDF/Mesure de Paris.pdf]

SCHABOL Roger (abbé), La théorie et la pratique du jardinage et de l'agriculture, par principes et démontrées d'après la physique des végétaux, Paris $1767,531 \mathrm{p}$.

SCHWEITZER Florence, Montreuil (Seine-Saint-Denis), 25-31 rue Ernest Savart, fouilles archéologiques 2 mai-6 juillet 2001, Document final de synthèse, INRAP, Pantin, 2002, 50 p. ; annexes et planches. 